\title{
Mathematical study on the thickened interface model by viscosity solution of the level-set equation
}

\author{
Nobuyuki OSHIMA* \\ ${ }^{*}$ Faculty of Engineering, Hokkaido University \\ Kita-13, Nishi-8, Nishi-ku, Sapporo, Hokkaido 060-8628, Japan \\ E-mail: oshima@eng.hokudai.ac.jp
}

Received: 11 December 2021; Revised: 12 January 2022; Accepted: 17 January 2022

\begin{abstract}
The level-set approach extended for its viscosity solution is investigated to derive a relation to the conservation law of fluid phenomena and the phase-field approach based on the free energy theory. This mathematical approach is useful to consider approximate models for fluid interface problems. Here, this approach is applied to the thickened interface model of the combustion flame problem to derive a new mathematical formulation by the viscosity solution of the level-set equation, which can maintain a flame propagation speed of global solution. This approach reveals a mathematical incompatibility of empirical thickened flame models and proposes some improvements.
\end{abstract}

Keywords : Level-set equation, Viscosity solution, Combustion flame, Thickened interface model, Conservation law, Phase field approach

\section{Motivation}

Flame is a primal issue in combustion theory, which gives not only visual images of complicated combustion phenomena but also mathematical approaches to analyze these phenomena. The combustion flame is typically characterized as a thin sheet-like structure similar to other fluid interface phenomena such as gas-liquid interfaces and shockwaves. The level-set equation is a useful mathematical tool to describe the spatial propagation of a surface and is a common numerical model for thin sheet-like structures in various interface phenomena (Osher et al., 2002); it also has been investigated in detail in mathematical analyses (Giga et al., 2002). The concept of the viscosity solution introduced by Chen et al. (1991) confirmed the uniqueness for the mean curvature flow equation. This concept leads to a basement of the re-initialization procedure (Russo et al., 2000; Olsson et al., 2005) for suppressing numerical instability of the level-set equation. Ishida et al. (2009) have verified a one-dimensional Allen-Cahn type equation, the most familiar model of the phase field approach, essentially to the same solution. Using an analogy of the two interface models by the level-set and phase field approaches, Liu and Oshima (2011) proposed a numerical model for a premixed combustion flame. Oshima $(2016,2017)$ later extended this model to a three-dimensional formultion for a viscosity solution of the level-set equation.

Because of the thin sheet-like structure of a combustion flame, its numerical simulation requires a fine grid resolution, which may become too small to solve even compared with the resolution required for a flow field. An artificially thickened interface approach (Legier et al., 2000; Collin et al., 2000; Durand and Polifke, 2007; Terashima et al., 2021) is useful for analyzing global flame behavior typically described by the burning velocity. Although their previous approaches have been successfully applied to a one-dimensional solution for the plane flame propagation of premixed combustion, solving multidimensional problems with a flame curvature remains problematic. These previous studies likely paid less attention to investigating the motive source by the flame curvature.

Essentially the same problem of a thickened interface has also been investigated at a liquid-gas phase interface. Tiwari et al. (2013) numerically solved this problem as a transport equation with a discontinuous solution. For the profile in the interface thickness, Nyigizente et al. (2021) mathematically analyzed a consistent formulation of the thickened phase interface to satisfy its global behavior as a force balance of surface tension and a theoretical condition of thermal 
variables. They gave appropriate solutions for steady and unsteady interface phenomena with no phase change or a slow phase changed compared with the flow and thermal fields. The combustion flame, however, has an additional physics induced by a large propagation speed (burning velocity) and a complicated system of chemical reactions, although it has characteristics similar to those of the fluid interface governed by thermo-fluid conservation law under large density variation. This approach causes difficulty in treating the combustion flame by a consistent thermodynamic model, as applied to the phase interface by Nyigizente et al. (2021).

As an alternative approach, the level-set equation concept enables the flame to be treated as a global interface phenomenon using a common mathematical approach when essential quantities such as the burning velocity and thickness are known to govern the combustion flame behavior. Oshima (2017) derived the extended level-set equation to its viscosity solution, which mathematically corresponds to the time-space differential equation of the conservation law and the phase field. In the present study, the extended level-set equation is applied to the thickened-interface approach to investigate its mathematically consistent formulation in the three-dimensional propagation.

\section{Consistent formulation of thickened interface}

\subsection{Formulation of level-set equation extended to the viscosity solution}

An original formulation of the level-set approach is expressed as

$$
\frac{\partial \phi}{\partial t}=v|\nabla \phi|
$$

where a level $\phi=\phi_{0}$ defines a surface shape and its temporal behavior with the constant $\phi_{0}$ calculated by a propellant speed $v$ normal to the surface element. In the formulation, a mean curvature $\kappa$ is defined by the normal unit vector $\mathbf{n}$ as $\kappa=\nabla \cdot \mathbf{n}, \mathbf{n}=\nabla \phi /|\nabla \phi|$. This type of equation is generally solved with additional diffusive terms or numerical errors to avoid an instability problem and to maintain solution uniqueness. Chen et al. (1991) and Ishida et al. (2009) verified mathematical fundaments for the viscosity solution of a level-set equation and for a one-dimensional Allen-Cahn type equation of the phase-field approach, respectively. Re-initialization procedures often introduced into numerical simulations (Russo et al., 2000; Olsson et al., 2015, 2017) express this concept to give a viscosity solution for this purpose.

Oshima $(2016,2017)$ applied this approach to a physical fluid interface with a large density variation governed by the thermal-fluid conservation law. An extended level-set equation computed to its viscosity solution is expressed as

$$
\rho \frac{\partial \phi}{\partial t}+\rho \mathbf{V} \cdot \nabla \phi=\rho v|\nabla \phi|+O(\phi, \nabla \phi) \text {. }
$$

Here, the variable $\phi$ is redefined as a convective scalar in fluid motived by material velocity vector $\mathbf{V}$ and fluid density $\rho$ and a propellant mass flux $\rho v=s+c \kappa$ is introduced, which is divided into two values of normal propagation $s$ and curvature flow $c \kappa$ of the level surface.

Instead of being modeled as a single level surface as defined by the original level-set approach, the interface is modeled by Eq. (2) as a finite thickness zone of laminated layers by multiple contours of levels $\phi=\phi_{0}$ $\left(\varphi_{-} \leq \varphi_{0} \leq \varphi_{+}\right)$, as shown in Fig. 1. If each contour has a continuous moving speed relative to its neighbors, the variable $\phi$ can form a local profile around the contour $\phi=\phi_{0}$. Thus, Eq. (2) gives a viscosity solution of level-set equation as laminated multiple contours in a finite thickness range $\left(\varphi_{-} \leq \varphi_{0} \leq \varphi_{+}\right)$. Functional $O(\phi, \nabla \phi)$ on the right-hand side of Eq. (2) defines an interaction between the neighboring levels to maintain their monotonic lamination mathematically, which may describe a physical model to maintain a stable thin interface zone. Differential-equationbased re-initialization methods (Russo et al., 2000; Olsson et al., 2015, 2017) are candidates for this functional model that has a stable zero solution in the time marching calculation by $\partial \phi / \partial t=O(\phi, \nabla \phi)$. Under the condition of $O(\phi, \nabla \phi)=0$, each level surface has the same propellant mass flux $\rho v=s+c \kappa$ so that the extended equation (2) becomes equivalent to the original level-set approach with the re-initialization procedure. 

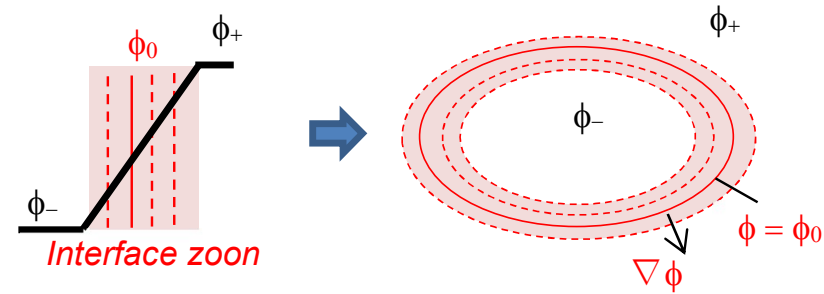

Fig. 1 Level-set approach extended by laminated multiple contours (Oshima, 2017).

Following Liu and Oshima (2011), the physical behavior of an interface were considered here on the basis of a relation to the scalar conservation law expressed by

$$
\rho \frac{\partial \phi}{\partial t}+\rho \mathbf{V} \cdot \nabla \phi=\nabla \cdot \mathbf{j}+q
$$

where the variable $\phi$ is a conservative scalar in a fluid motived by convection flux and diffusion flux by the gradient model as $\mathbf{j}=d \nabla \phi$ (positive diffusion factor: $d>0$ ) and source $q$, respectively. To derive the level-set formulation from the conservation equation (Eq. (4)), Liu et al. (2011) introduced a variable propellant speed $s^{*}(\phi)=q /|\nabla \phi|$ (physically defined as a local burning velocity or local flame speed) that describes both the global propellant speed $S$ and the local stable solution of flame steepness $|\nabla \phi|$ observed at the diffusion-neutral position $(\nabla \cdot \mathbf{j}=0)$.

Oshima (2017) proposed an extended formulation to the three-dimensional problem with the surface curvature flow as

$$
q=-\left(\rho v+f^{\prime}\right) \frac{f}{d}+\kappa f, \quad f^{\prime}=\frac{\mathrm{d} f}{\mathrm{~d} \phi} .
$$

Here, the propellant flux $\rho v=s+c \kappa$ gives a global interface behavior in the same manner as in Eq. (2) and a shape function $f(\phi)$ is introduced to define a profile of $\phi$ through the flame thickness under the following condition:

$$
\frac{f}{d}+|\nabla \phi|=0 \text {. }
$$

Under the assumption of a parabolic function for $f(\phi)$, Inage et al. (2007) proposed the same formulation without curvature flow terms proportioned to $\kappa$ in Eq. (4) as a hyperbolic tangent approximation (HTA) model for a combustion flame. Upon substitution of Eq. (4), the right-hand side of conservative Eq. (3) can be re-written with the diffusion term as

$$
R H=\rho v|\nabla \phi|-\left(\rho v+f^{\prime}\right)\left(\frac{f}{d}+|\nabla \phi|\right)+\nabla \cdot d\left(\frac{f}{d}+|\nabla \phi|\right) \mathbf{n},
$$

which gives a viscosity solution of the level-set equation in Eq. (2) when the second and third terms disappear in the thickness profile of Eq. (5). As Oshima (2017) analyzed, the role of the third term is re-initialization converging the thickness profile solution by Eq. (12) in the interface. We also expected that the second term, $\rho v=0$, to give a converging solution around the contour surface at $f^{\prime}=0$, which might be located at the steepest gradient $|\nabla \phi|$. Such formulations have been proposed by other researchers (Russo et al., 2000; Olsson et al., 2005) as re-initialization procedures of the level-set equation.

Equation (4) for the interface motive force in the level-set solution can also be derived by a phase-field approach based on the free energy theory. The interface motive force in the Allen-Cahn equation is formulated by a Euler-Lagrange equation,

$$
\frac{D \phi}{D t} \equiv \frac{\partial \phi}{\partial t}+\mathbf{V} \cdot \nabla \phi=-M \sum \frac{\delta \Phi_{i}}{\delta \phi}
$$

by the variational of functional values $\Phi_{i}$ to the variable $\phi$, which is often called as an "order parameter" in the phasefield approach and corresponds to the level-set function indicating the interface position. Functional (8) is individually evaluated around each laminated contour, and the mobility factor $M$ in Euler-Lagrange Eq. (7) is given by $\rho d M=1$ to maintain an equivalent variation $d|\nabla \phi|$ throughout the integration thickness. 
A functional value $\Phi_{i}$ is defined as

$$
\Phi_{i}=\int F_{i} \mathrm{~d} V
$$

by free energy potentials $F_{i}$ describing the $i$-th elemental effect integrated through the volume $V$ of an interface element.

In the present research, the potential functions are given by four components as

$$
F_{1}=\int s f \mathrm{~d} \phi, F_{2}=\frac{1}{2} f^{2}, F_{3}=(d-c) f|\nabla \phi|, F_{4}=\frac{1}{2} d^{2}|\nabla \phi|^{2},
$$

where $F_{2}$ and $F_{4}$ are often applied in the phase-field approach to local equilibrium interface problems when $F_{2}$ is a double-well function of variable $\phi$, a most simple formula of which is defined by the parabolic function $f$ in Olsson's re-initialization. $F_{1}$ models an interface propellant in nonequilibrium interface problems (Kobayashi, 1993). $F_{3}$, newly proposed by Oshima (2017), considers a local nonequilibrium due to the previously discussed laminated neighboring layers, which induces a curvature flow of $c \kappa$. Notably, $F_{3}=0$ by $c=d$ induces a "nonzero curvature flow" proportioned to the diffusion coefficient $d$. Solving multidimensional problems with an interface curvature is difficult, although Inage et al. (2007) and Kobayashi (1993) devoted some attention to this problem.

\subsection{Transport equation accompanied by level-set solution}

When a double-welled function $F_{2}$ is given by a square of shape function $f$, Eqs. (3) and (4) (or its equivalent Eq. (7)) gives a monotonic solution of $\phi$ along the coordinate $\xi$ normal to the interface, where Eq. (5) is expressed as

$$
f=-d|\nabla \phi|=-d(\mathbf{n} \cdot \nabla \phi)=-d \frac{\mathrm{d} \phi}{\mathrm{d} \xi} .
$$

Thus, any continuous profile along the coordinate $\xi$ can be defined by a function $\phi_{a}(\phi)$ that is not required to be monotonic or positive, although the interface level-set variable $\phi$ should be monotonic. We now introduce a new function $f_{a}(\phi)$ and its derivative of $\phi$ along the interface normal coordinate $\xi$ as

$$
f_{a}=-d\left(\mathbf{n} \cdot \nabla \phi_{a}\right)=-d \frac{\mathrm{d} \phi_{a}}{\mathrm{~d} \xi}=\phi_{a}^{\prime} f, \text { where } \phi_{a}{ }^{\prime}=\frac{\mathrm{d} \phi_{a}}{\mathrm{~d} \phi},
$$

and

$$
f_{a}^{\prime}=\frac{\mathrm{d} f_{a}}{\mathrm{~d} \phi}=\phi_{a}^{\prime} f^{\prime}+\phi_{a}^{\prime \prime} f, \text { where } \phi_{a}^{\prime \prime}=\frac{\mathrm{d}^{2} \phi_{a}}{\mathrm{~d} \phi^{2}},
$$

though they are not generally defined as functions of $\phi_{a}$.

Following the previously discussed re-initialization approach, the following equation for $\phi_{a}$ is derived as

$$
\rho \frac{\partial \phi_{a}}{\partial t}+\rho \mathbf{V} \cdot \nabla \phi_{a}=\rho v \mathbf{n} \cdot \nabla \phi_{a}-\left(\rho v \phi_{a}{ }^{\prime}+f_{a}{ }^{\prime}\right)\left(\frac{f}{d}+\mathbf{n} \cdot \nabla \phi\right)+\nabla \cdot d\left(\frac{f_{a}}{d}+\mathbf{n} \cdot \nabla \phi_{a}\right) \mathbf{n}
$$

where the propellant flux $\rho v=s+c \kappa$ and the normal unit vector $\mathbf{n}$ are the same as those in Eq. (6) for the level-set variable $\phi$. Like Eq. (6), Eq. (13) propagates a solution of $\phi_{a}$ accompanied by the interface propellant flux by $\rho v$ when both conditions for the stable solutions of $\phi$ and $\phi_{a}$ are given by Eqs. (10) and (11), respectively. Notably, Eq. (13) for $\phi_{a}$ is accompanied by the level-set solution of $\phi$ by Eqs. (3) and (6) with the normal unit vector $\mathbf{n}$ and mean curvature $\kappa$ of a thin interface and its condition in Eq. (5), which diminishes the second term in the right-hand side. On the contrary, the last term of Eq. (13) has a role of re-initialization:

$$
\frac{f_{a}}{d}+\mathbf{n} \cdot \nabla \phi_{a}=0
$$

In the same manner used to derive the relation between Eqs. (4) and (6), Eq. (13) can be transformed into an equivalent formulation describing the conservation law as

$$
\rho \frac{\partial \phi_{a}}{\partial t}+\rho \mathbf{V} \cdot \nabla \phi_{a}=\nabla \cdot \mathbf{j}_{a}+q_{a}, \quad \mathbf{j}_{a}=d \nabla \phi_{a}, \quad q=-\left(\rho v \phi_{a}{ }^{\prime}+f_{a}{ }^{\prime}\right) \frac{f}{d}+f_{a} \kappa,
$$

where an identity of the vector formula and derivative relation are applied as

$$
\nabla \cdot f_{a} \mathbf{n}=f_{a}{ }^{\prime} \mathbf{n} \cdot \nabla \phi+f_{a} \kappa \text { and }\left(\mathbf{n} \cdot \nabla \phi_{a}\right) \mathbf{n}=\frac{\mathrm{d} \phi_{a}}{\mathrm{~d} \phi}(\mathbf{n} \cdot \nabla \phi) \mathbf{n}=\frac{\mathrm{d} \phi_{a}}{\mathrm{~d} \phi} \nabla \phi=\nabla \phi_{a} .
$$


By substituting Eqs. (11) and (12), another formation of source term of Eq. (15) is derived as

$$
q=-\left(s+f^{\prime}\right) \frac{f_{a}}{d}-(c-d) \kappa \frac{f_{a}}{d}-\phi_{a} " \frac{f^{2}}{d},
$$

which consists of three motive forces: the first term by Liu's variable propellant speed $s^{*}=s+f^{\prime}$, the second term by the curvature flow source by $d \kappa$ against the curvature component of the diffusion term, and a third force driven by the interface shape function $f$. Here, the first and second forces and the diffusion flux $\mathbf{j}_{a}=d \nabla \phi_{a}$ give a common formula $\left(s^{*}+c \kappa\right) \mathbf{n} \cdot \nabla \phi_{a}$ under the stable solution by Eq. (14). By contrast, the third force, which is evaluated as $\phi_{a} " f|\nabla \phi|$ maintains an individual profile of targeting solution $\phi_{a}(\phi)$, accompanied by the global interface defined by the shape function $f$ and $|\nabla \phi|$. Therefore, it is a mathematically consistent formulation for the stable profile variables in the propellant interface when it is governed by the conservation law with convection and diffusion motivation.

\subsection{Consistent formulation of thickened interface}

In the last section, a common formulation (Eq. (13) or (15)) was derived for the variables that accompany the stably propellant interface. This derivation has both good and bad news for previous investigations of thickened interface models: the present formulation (15) confirms the idea of previous thickened models inducing a reciprocal factor of diffusion coefficient $d$ to the source term that gives a correct solution for the plane interface without the curvature, whereas it indicates that such a solution should also be deformed by the curvature flow because of the modified diffusion coefficient $d$ in general multidimensional problems. This result means that any formulation without the mean curvature cannot give the correct solution based on this idea of applying a thickening interface via a modified diffusion coefficient.

If the profile $\phi_{a}(\phi)$ or its shape function $f_{a}(\phi)$ is known, Eq. (13) or (15) can be applied to the solution with the correct mean curvature flow. Therefore, a modified formulation for a thickened interface is naturally derived by introducing a stretching factor $\alpha$ to the diffusion coefficient $d$ as

$$
\rho \frac{\partial \phi_{a}}{\partial t}+\rho \mathbf{V} \cdot \nabla \phi_{a}=\rho v \mathbf{n} \cdot \nabla \phi_{a}-\left(\rho v \phi_{a}{ }^{\prime}+f_{a}{ }^{\prime}\right)\left(\frac{f}{\alpha d}+\mathbf{n} \cdot \nabla \phi\right)+\nabla \cdot \alpha d\left(\frac{f_{a}}{\alpha d}+\mathbf{n} \cdot \nabla \phi_{a}\right) \mathbf{n},
$$

which should be solved together with the interface level-set variable as

$$
\rho \frac{\partial \phi}{\partial t}+\rho \mathbf{V} \cdot \nabla \phi=\rho v \mathbf{n} \cdot \nabla \phi-\left(\rho v+f^{\prime}\right)\left(\frac{f}{\alpha d}+\mathbf{n} \cdot \nabla \phi\right)+\nabla \cdot \alpha d\left(\frac{f}{\alpha d}+\mathbf{n} \cdot \nabla \phi\right) \mathbf{n} .
$$

Following the previous discussion, the identical formations of the conservation law are also derived as

$$
\rho \frac{\partial \phi_{a}}{\partial t}+\rho \mathbf{V} \cdot \nabla \phi_{a}=\nabla \cdot \alpha \mathbf{j}_{a}+Q_{a}, \quad Q_{a}=-\left(\rho v \phi_{a}{ }^{\prime}+f_{a}{ }^{\prime}\right) \frac{f}{\alpha d}+f_{a} \kappa
$$

and

$$
\rho \frac{\partial \phi}{\partial t}+\rho \mathbf{V} \cdot \nabla \phi=\nabla \cdot \alpha \mathbf{j}+Q, \quad Q=-\left(\rho v+f^{\prime}\right) \frac{f}{\alpha d}+f \kappa .
$$

Here, the new profiles $\phi$ and $\phi_{a}(\phi)$ are defined by

$$
\frac{f}{\alpha d}+\mathbf{n} \cdot \nabla \phi=0 \quad \text { and } \quad \frac{f_{a}}{\alpha d}+\mathbf{n} \cdot \nabla \phi_{a}=0 .
$$

However, these profiles are generally not applicable because $f_{a}$ may be unknown before the model equation is solved. Thus, the last term in Eq. (17) can be replaced with $-\alpha d \kappa \mathbf{n} \cdot \nabla \phi_{a}$ as an assumption of a locally steady solution. This approach can lead to a practical improvement for the curvature flow problem in the previous flame thickening models as

$$
Q_{a}=\frac{q_{a}}{\alpha}+(1-\alpha) d \kappa \mathbf{n} \cdot \nabla \phi_{a}
$$

where the interface level-set variable $\phi$ is not needed because it is removed from the explicit formulation of $\phi_{a}$.

An alternative proposal is to keep the same diffusion coefficient $d$, which might induce curvature flow in most physical problems. When the curvature flow is given by $d$ as $c=d$, the source term $q_{a}$ has no contribution from the interface curvature $\kappa$. Assuming the stable solution by Eq. (14) and the known propellant speed $S$, the source term is decomposed into propellant and residual terms as 


$$
q_{a}=s \mathbf{n} \cdot \nabla \phi_{a}+q_{a}^{*}, \quad q_{a}^{*}=-\frac{1}{d}\left(\phi_{a}^{\prime} F_{2}{ }^{\prime}+2 \phi_{a}^{\prime \prime} F_{2}\right), \quad F_{2}{ }^{\prime}=\frac{\mathrm{d} F_{2}}{\mathrm{~d} \phi} .
$$

Here, $q_{a}^{*}$ depends only on the shape function as $2 F_{2}=f^{2}$ in Eq. (9) when the variable $\phi_{a}(\phi)$ maintains an identity in the thickened interface. Therefore, a constant factor $\alpha$ can modify the shape function $f / \alpha$, giving a corresponding formulation of $q_{a}^{*}$ to satisfy the thickened interface conditions defined in Eq. (21).

Finally, a new model is proposed for the thickened interface as

$$
\rho \frac{\partial \phi_{a}}{\partial t}+\rho \mathbf{V} \cdot \nabla \phi_{a}=\nabla \cdot \mathbf{j}_{a}+Q_{a}, \quad \mathbf{j}_{a}=d \nabla \phi_{a}, \quad Q_{a}=s \mathbf{n} \cdot \nabla \phi_{a}+\frac{q_{a}^{*}}{\alpha^{2}}=\left(1-\frac{1}{\alpha}\right) s \mathbf{n} \cdot \nabla \phi_{a}+\frac{q_{a}}{\alpha^{2}}
$$

and

$$
\rho \frac{\partial \phi}{\partial t}+\rho \mathbf{V} \cdot \nabla \phi=\nabla \cdot \mathbf{j}+Q, \quad \mathbf{j}=d \nabla \phi_{a}, \quad Q=\left(1-\frac{1}{\alpha}\right) s|\nabla \phi|+\frac{q}{\alpha^{2}},
$$

where thickened solutions of $\phi_{a}$ and $\phi$ satisfying the targeted condition (Eq. (21)) are propelled with the consistent interface flux $\rho v=s+d \kappa$. Equation (24) appears to be an available formulation for the combustion flame because it does not require preliminary information of shape function $f_{a}$ or $f$ except for the interface flux vector $s \mathbf{n}$. Here, the thickened solutions $\phi_{a}$ and $\phi$ keep the same relation $\left(\mathrm{d} \phi_{a} / \mathrm{d} \phi\right)$ under the above assumption as the relation in the original solutions. Thus, profiles $\phi_{a}$ can be solved along the unique progress variable $\phi$ with a thickened profile in the physical space.

\subsection{Phase-field approximation of a thickened interface}

The phase-field approach is useful for considering the physical mechanics of interfacial phenomena based on thermodynamics theory. Following Oshima (2017), the thickened interface (Eq. (20)) of level-set variable $\phi$ is translated to the potential formulation of the Allen-Cahn equation by inducing the large diffusion coefficient $\alpha d$ in the third and fourth potential components as

$$
F_{3}=(\alpha d-c) f|\nabla \phi|, F_{4}=\frac{1}{2}(\alpha d)^{2}|\nabla \phi|^{2},
$$

where the mobility factor should be modified in the same manner as $\rho(\alpha d) M=1$. Thus, the component $F_{3}$ is necessary to maintain the identity of interface propellant flux $\rho v=s+c \kappa$ even if $F_{3}=0$ in the physical condition. Because of this common thermal mechanics of the interface, the diffusion control approach for thickened flame models needs to introduce a compensating treatment for the curvature flow due to diffusion flux, as discussed by Nyigizente et al. (2021) for the gas-liquid phase interface.

On the contrary, the alternative formulation (Eq. (25)) is also derived by inducing factor $\alpha$ to the shape function $f$ in Eq. (9) as

$$
F_{1}=\int s\left(\frac{f}{\alpha}\right) \mathrm{d} \phi, F_{2}=\frac{1}{2}\left(\frac{f}{\alpha}\right)^{2}, F_{3}=(d-c) \frac{f}{\alpha}|\nabla \phi|, F_{4}=\frac{1}{2} d^{2}|\nabla \phi|^{2} .
$$

where the third component $F_{3}=0(c=d)$ and the mobility factor $\rho d M=1$ keep the identity of the interface propellant flux $\rho v=s+c \kappa$. This approach appears to be simpler than the other for the combustion flame problem, which includes a complicated diffusion mechanism when the chemical reaction has a known contribution to propellant flux $\rho v=s+c \kappa$ as the flame burning velocity $s$ under the aforementioned assumption $c=d$.

Finally, in the same manner, the potential formulation of the accompanying variable $\phi_{a}$ to the phase-field approach is derived as

$$
F_{1}=\int s f_{a} \mathrm{~d} \phi, F_{2}=\int\left(f_{a}^{\prime} f\right) \mathrm{d} \phi, F_{3}=(\alpha d-c) f_{a}|\nabla \phi|, F_{4}=\frac{1}{2}(\alpha d)^{2}|\nabla \phi|^{2}, \rho(\alpha d) M=1,
$$

and

$$
F_{1}=\int s\left(\frac{f_{a}}{\alpha}\right) \mathrm{d} \phi, F_{2}=\int \frac{f_{a}^{\prime} f}{\alpha^{2}} \mathrm{~d} \phi, F_{3}=(d-c) \frac{f_{a}}{\alpha}|\nabla \phi|, F_{4}=\frac{1}{2} d^{2}|\nabla \phi|^{2}, \quad \rho d M=1,
$$

which correspond to Eqs. (26) and (27), respectively. These equations give the same solution, of course, as the physical solution with $\alpha=1$. Notably, however, the potential formulation in this case is a consistent mathematical model but is 
not necessary to give a free energy in the physical phenomena because the mobility factor $M$ has not yet been sufficiently physically investigated for the mechanics of a laminated interface. This topic is left for future works, especially works on combustion flames whose mobility may have a substantial nonlinear relation to the variable representing the interface level-set, similar to the relation of the well-known Arrhenius law to temperature.

\section{Numerical results}

The aforementioned mathematical study on the thickened interface approach derives a consistent equation system based on the viscosity solution of the level-set equation and its equivalent formulation of the conservation law. The proposed approach may solve a difficulty encountered in the thickened interface models which implicitly includes the curvature flow due to a diffusion term. In this section, a few examples are presented of one-dimensional solutions obtained using the newly proposed method applied to numerical simulations.

As a simplified problem, the interface level-set $\phi$ is solved by the shape functions $f=\phi(\phi-1)$, where the stable profile of Eq. (10) along the interface normal coordinate $\xi$ is calculated as

$$
\phi=\frac{1}{2}+\frac{1}{2} \tanh \left(\frac{\xi}{2 d}\right) \quad 0 \leq \phi \leq 1 .
$$

When an accompanying variable $\phi_{a}=(A \phi+B) \phi(1-\phi)$ is assumed, its shape function is calculated as

$$
f_{a}=\phi_{a}{ }^{\prime} f=\left\{A(1-2 \phi)+B\left(2 \phi-3 \phi^{2}\right)\right\} \phi(\phi-1)
$$

under conditions (11). In the present study, the factors are set as $A=1$ and $B=-1.5$ to create a nonmonotonic profile that gives $\phi_{a}$ with $\phi$ in Eq (26) at the initial condition. Two cases are evaluated by the plane interface coordinate $x=\xi$ and the cylindrical interface coordinate $r=\xi$, where the interface curvature is $\kappa=0$ and $\kappa=1 / r$, respectively. The interface propellant factors are given as $s=1$ or 0 and $c=d$ or 0 , whereas the diffusion coefficient is set as $d=0.2$. The density is assumed to be constant at $\rho=1$. As mentioned above, a propellant mass flux $\rho v=s+c \kappa$ is defined by the normal propagation $s$ and curvature flow $c \kappa$ of the level surface.

A system of differential equations (Eqs. (19) and (20)) for thickened factor $\alpha$ is solved by the Euler explicit algorithm for the time integration and the second-order central difference scheme for the spatial derivatives. The same solution is exactly given by the equivalent formulations, Eqs. (17) and (18). The explicit diffusion term in the conservation formula introduced by the viscosity solution approach can avoid an additional artificial procedure or upwind scheme for removing numerical instability. Time and spatial increments are set as $\mathrm{d} t=0.01$ and $\mathrm{d} \xi=0.1$ to satisfy the conditions

$$
v \frac{\mathrm{d} t}{\mathrm{~d} \xi}<1, \frac{d}{\rho} \frac{\mathrm{d} t}{\mathrm{~d} \xi^{2}}<\frac{1}{2} \text { and } \frac{v}{d} \mathrm{~d} x<2
$$

in the same manner as in the convection and diffusion problems.

The first case for the original plane interface $(\alpha=1)$ gives a uniformly propellant profile by the speed factor $s=1$ both for the level-set variable $\phi$ and the accompanying variable $\phi_{a}$ in Fig. 2, where the curvature factor $c$ does not contribute to the solution. When a thickened factor $(\alpha=2)$ is applied, the solutions shown in Fig. 3 become the thickened profile, which is extended to the $x$-coordinate with the correct factor to the original profiles in Fig. 3 and is propelled by the same speed.

The second case for the cylindrical interface with the same thickened factor $(\alpha=2)$ is operated by the speed factor $s=0$ to evaluate the curvature flow. When the curvature factor is set equal to the diffusion coefficient as $c=d=0.2$, which corresponds to the thickened interface model without the correction of curvature flow by the diffusion term, the solution (fig.4 left) is gradually deformed from the initial profile. On the contrary, the zero curvature factor $c=0$ corrects the solution (fig. 4 right), which maintains the same initial profile defined by Eq. (21). 


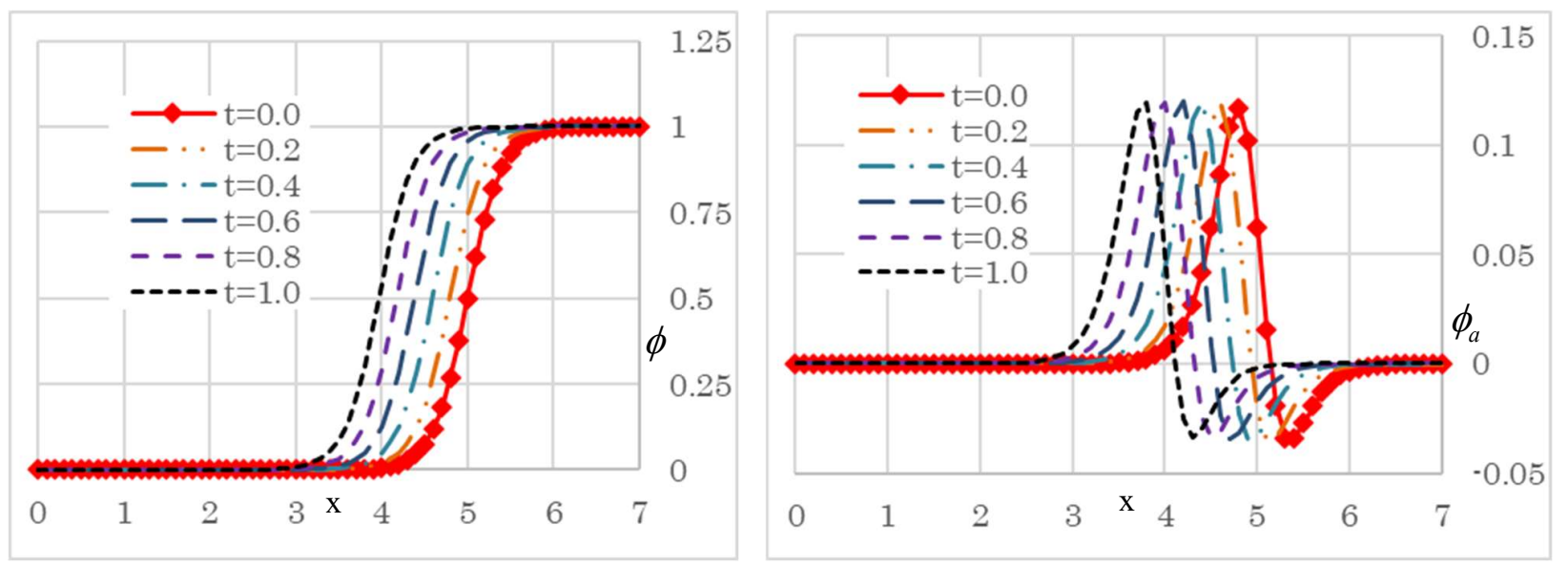

Fig.2 The plane interface solutions of the level-set variable $\phi$ (left) and the accompanying variable $\phi_{a}$ (right); the original profiles $(\alpha=1)$ under the conditions $s=1$ and $c=d=0.2$.
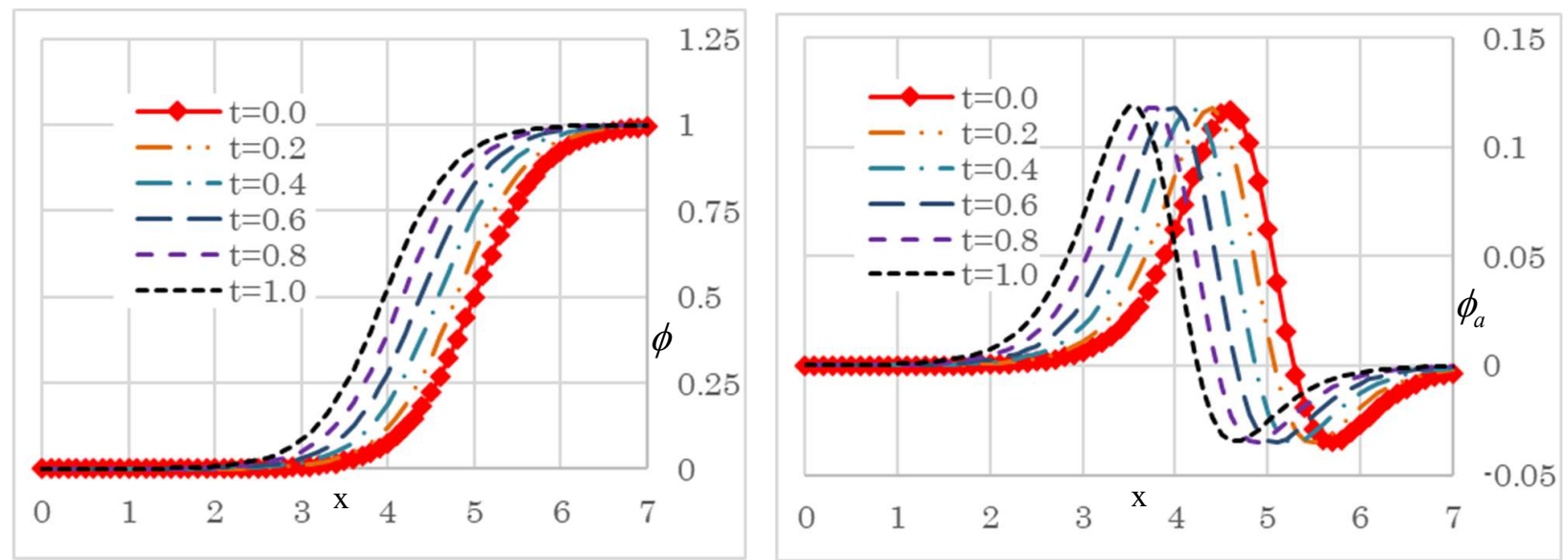

Fig.3 The plane interface solutions of the level-set variable $\phi$ (left) and the accompanying variable $\phi_{a}$ (right); the thickened profiles $(\alpha=2)$ under the conditions $s=1$ and $c=d=0.2$.
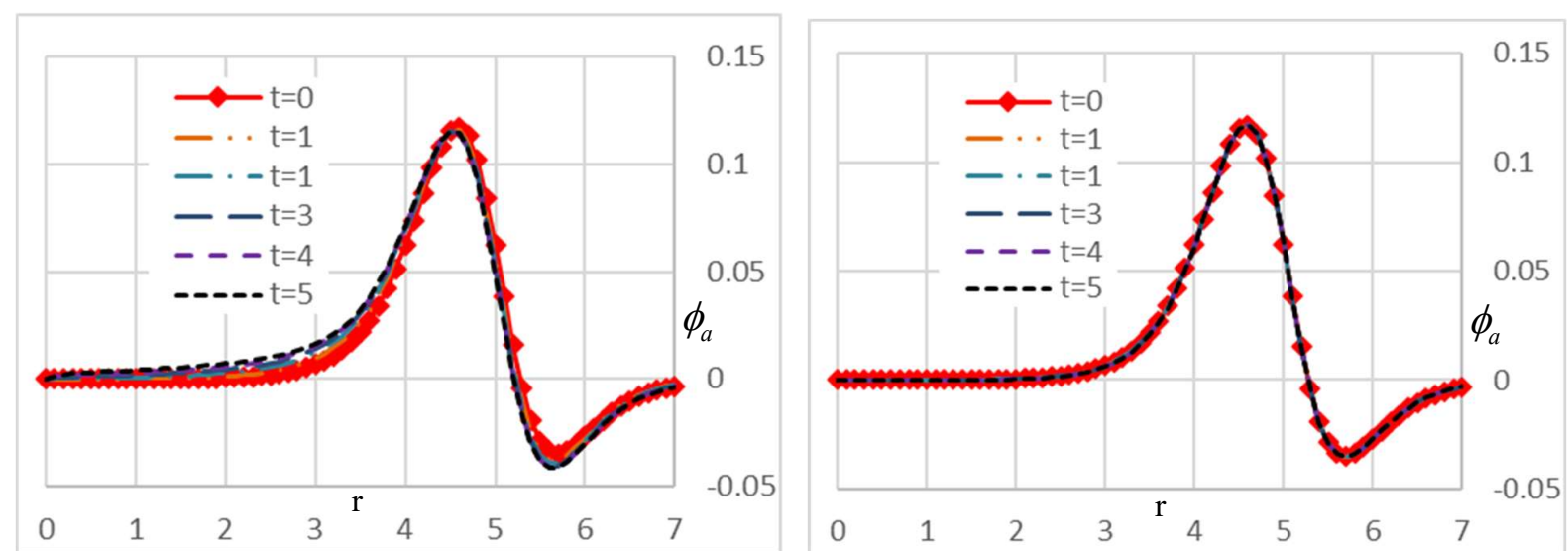

Fig.4 The cylindrical interface solutions of $\phi_{a}$; the thickened profiles $(\alpha=2)$ under the conditions $s=0$ and $c=d=0.2$ (left) and $c=0, d=0.2$ (right) 


\section{Conclusion}

In this paper, we investigated the mathematically consistent formulation for the thickened interface model based on the viscosity solution of the level-set equation. It led to three major proposals:

1) A new mathematical model is introduced for the variable transported in the stably propellant interface as the accompanying equation of the interface level-set. It can predict the stable profile solution of the conservation law with the given interface propellant flux.

2) A mathematically consistent formulation is derived for the thickened interface model to treat solve the interface curvature contribution correctly to overcome a difficulty encountered in the previous models for multidimensional problems.

3) A potential formulation for a phase-field approach is also derived to explain the physical mechanics driving the aforementioned new model in the combustion flame and gas-liquid phase interface.

\section{References}

Chen, Y., Giga, Y. and Goto, S., Uniqueness and existence of viscosity solution of generalized mean curvature flow equations, Journal of Differential Geometry, Vol.33 (1991), pp. 749-786. DOI: 10.4310/jdg/1214446564.

Colin, O, Ducros, F., Veynante, D. and Poinsot, T., A thickened flame model for large eddy simulations of turbulent premixed combustion, Physics of Fluids, Vol.12, No.7 (2000), pp.1843-1863. DOI: 10.1063/1.870436.

Giga, Y., Surface evolution equations - a level set method, Hokkaido University Technical Report Series in Mathematics, (2002). DOI: 10.14943/647.

Inage, A., Proposal of United Combustion Model for Premixed and Diffusion Flames and Its Evaluation (1st Report: Estimation of the Fundamental Model), Journal of the Combustion Society of Japan, Vol.49. No.150 (2007), pp.267276, in Japanese. DOI: 10.1115/GT2008-50233.

Ishida, A. and Tonegawa, Y., A relation between a mean curvature equation and a phase transform of parabolic equations in an equation for the premixed combustion flames, Proceedings of Annual Meeting of Mathematical Society of Japan (2009) 2p, in Japanese.

Kobayashi, R., Modeling and numerical simulations of dendritic crystal growth, Physica D, Vol.63 (1993), pp.410-423. DOI: 10.1016/0167-2789(93)90120-P.

Legier, J.P., Poinsot, T. and Veynante, D., Dynamically thickened flame LES model for premixed and non-premixed turbulent combustion, Proceedings of the Summer Program 2000, Center for Turbulence Research, (2000), pp.157168.

Liu, Y. and Oshima, N., A level set approach for a premixed flame based on a new concept of flame speed, Journal of Thermal Science and Technology, Vol.6, No.1 (2011), pp. 140-153. DOI: 10.1299/jtst.6.140.

Nyigizente, D., Ducruix S. and Schmitt, T., Development of an interface thickening method for the direct numerical simulation of compressible liquid-vapor flows in the framework of the second gradient theory, Physics of Fluids, Vol.33, 052119 (2021). DOI: 10.1063/5.004875.

Olsson, E. and Kreiss, G., A conservative level set method for two phase flow, Journal of Computational Physics, Vol.210 (2005), pp. 225-246. DOI: 10.1016/j.jcp.2005.04.007.

Osher, S. and Fedkiw, R., Level set methods and dynamic implicit surfaces, Springer (2002). ISBN-13: 978-0387954820.

Oshima, N., An extensional formulation for a diffusive solution of the level-set equation by considering a relation to the scalar conservation equation, Mechanical Engineering Letters, Vol.2 (2016), 16-00220. DOI: 10.1299/mel.16-00220.

Oshima, N., A fluid interface model by phase field approach applied to the diffusive solution of level-set equation, Mechanical Engineering Letters, Vol.3 (2017), 17-00080. DOI: 10.1299/mel.17-00080.

Russo, G. and Smereka, P., A remark on computing distance functions, Journal of Computation Physics, Vol.164 (2000), pp. 51-67. DOI: 10.1006/jcph.2000.6553.

Terashima H., Haneda, Y. and Kawai, S., A localized thickened flame model for simulations of propagation and autoignition under elevated pressure conditions, Proceedings of Combustion Institute, 38 (2021), pp.2119-2126. DOI: 10.1061/j.proci.2020.06.063.

Tiwari, A., Freund, J. B. and Pantano, C., A diffuse interface model with immiscibility preservation, Journal of Computational Physics, Vol.252 (2013), pp.290-309. DOI: 10.1016/j.jcp.2013.06.021. 